\title{
Caso práctico de valuación de una MIPYME del sector de manejo de residuos y desechos en México a través del método de las dos betas (A practical case of valuation applied to a SME of the sector of management of residues and wastes in Mexico through the method of the two betas)
}

\author{
Axel Rodríguez Batres \\ Edgar Mauricio Flores Sánchez*
}

\begin{abstract}
The sector in Mexico denominated as private non-financial services represents a field of great relevance for the national economic and social growth due to the important amount of jobs that it contributes. Specifically, the business support and waste management and remediation services subsector has presented in recent years a remarkable increase in the number of personnel employed. Subsequently, the branch corresponding to waste management and remediation services means an important area not only of economic and social concern but also for the environment. The traditional valuation methods used on real estate applies several innovative techniques that are just beginning to be used for the valuation of companies and its corresponding decision making. The method of valuation of the two Betas comes from the market approach and allows to obtain a precise result of the value of an asset or company from easily obtained data belonging to a sample of comparable elements. The results obtained allowed to apply this method to a case study and obtain its market value.
\end{abstract}

Key words: beta distribution function, private non-financial services, two betas method, valuation of companies, waste management and remediation services

JEL: C10, G10, Q50.

\footnotetext{
- Universidad Iberoamericana Puebla, Departamento de Negocios. Blvd. del Niño Poblano, No. 2901, Colonia Reserva Territorial Atlixcáyotl, San Andrés Cholula, Puebla., 72810. Correo electrónico: axel_rb@hotmail.com

- Universidad Iberoamericana Puebla, Departamento de Negocios. Blvd. del Niño Poblano, No. 2901, Colonia Reserva Territorial Atlixcáyotl, San Andrés Cholula, Puebla., 72810. Correo electrónico: e.mfs@hotmail.com
} 
Resumen. El sector en México denominado como servicios privados no financieros representa un campo de gran relevancia para el crecimiento tanto económico como social del país a causa de la importante cantidad de empleos que aporta. Específicamente, el subsector apoyo a los negocios y manejo de desechos y servicios de remediación ha presentado en los últimos años un incremento destacable en cuanto a la cifra de personal ocupado. Subsecuentemente, la rama correspondiente a manejo de desechos y servicios de remediación significa un área importante no solo por el aspecto económico y social sino también ambiental. La valuación tradicional de inmuebles utiliza diversos métodos innovadores que recién están comenzando a ser utilizados para la valuación de empresas y su correspondiente toma de decisiones. El método de valuación de las dos Betas proviene del enfoque de mercado y permite obtener un dato preciso del valor de un activo o empresa a partir de datos fáciles de obtener pertenecientes a una muestra de elementos comparables. Los resultados alcanzados permitieron aplicar dicho método a un caso de estudio y obtener su valor de mercado.

Palabras clave: función de distribución beta, manejo de desechos y servicios de remediación, método de las dos betas servicios privados no financieros, valuación de empresas

\section{Introducción}

Es debido a la propia naturaleza humana que aquellas personas que se identifican con el mismo tipo de objetivos personales se reúnan en grupos con el objetivo de alcanzar dichas metas de manera más efectiva, de esta forma se conforman las denominadas organizaciones. Dentro de ellas, existen algunas cuyo principal objetivo se circunscribe entorno a la maximización de los beneficios de sus promotores o inversionistas, a dichas organizaciones se les asigna el nombre de empresas. Las empresas son entonces, aquellas organizaciones que por medio de la búsqueda de objetivos privados, y por medio de la gestión de diversos recursos así como de personas, ofrecen al mercado un conjunto de bienes y/o servicios, que a su vez son demandados por la sociedad para la satisfacción de sus necesidades. De esta forma las empresas son las unidades económicas que efectivamente representan la fuerza de crecimiento para cualquier país, pues debido al impacto de sus operaciones, generan empleos de manera tanto directa como indirecta, proporcionan satisfactores para todo tipo de consumidores y además 
contribuyen con el pago de impuestos recaudados por los diferentes niveles de Gobierno.

EI PIB o Producto Interno Bruto es el indicador principal empleado para medir el crecimiento de una economía local, regional o mundial, está constituido básicamente por el conjunto del valor estimado en dinero de los bienes y servicios creados por la entidad en un periodo determinado. Específicamente, el (Banco de México, 2017) lo define como: "Es el valor total de los bienes y servicios producidos en el territorio de un país en un periodo determinado, libre de duplicaciones. Se puede obtener mediante la diferencia entre el valor bruto de producción y los bienes y servicios consumidos durante el propio proceso productivo, a precios comprador (consumo intermedio). Esta variable se puede obtener también en términos netos al deducirle al PIB el valor agregado y el consumo de capital fijo de los bienes de capital utilizados en la producción". Es por lo anterior que se considera que un incremento constante del PIB es un objetivo que las autoridades del Gobierno deben considerar de gran importancia, así como la generación de mecanismos que auténticamente reflejen dicho crecimiento en un aumento en el nivel y calidad de vida de los ciudadanos.

Después de elaborar un análisis de los principales sectores económicos en México (INEGI, 2014), se presenta a continuación una figura referente alas a personas ocupadas en cada uno (Figura 1).

Figura 1. Estructura de sectores en México por porcentaje de personas ocupadas por sector (porcentajes).

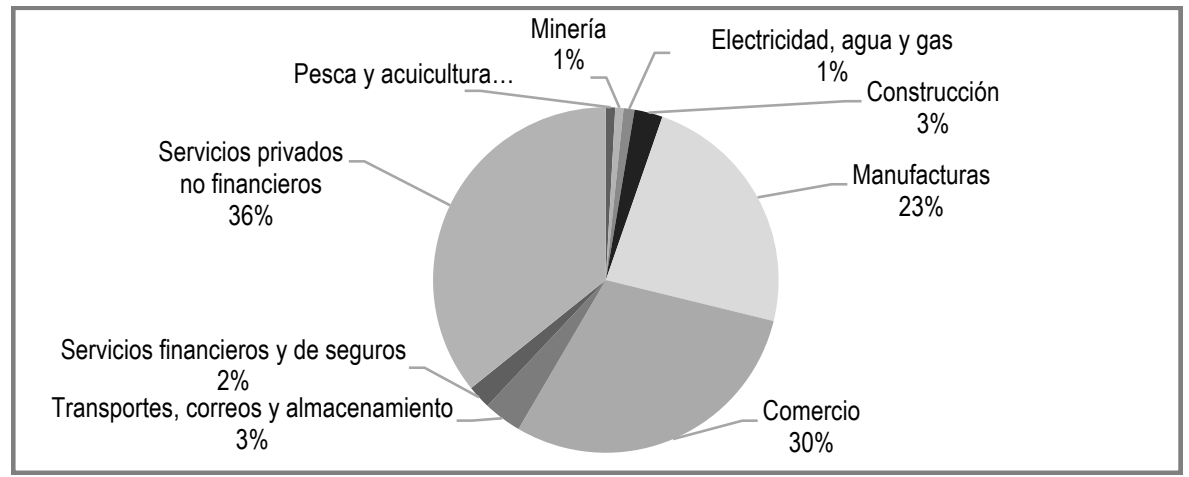

Fuente: Elaboración propia a partir de http://www.inegi.org.mx/est/contenidos/proyectos/ce/ce2014/default.aspx 
Como se puede observar en la Figura 1, es el sector de servicios privados no financieros aquél que reporta el más alto porcentaje de contribución al número de personas ocupadas en México. Adicionalmente, de acuerdo a información obtenida de (INEGI, 2014) la cantidad como porcentaje de aportación al valor total de la producción en México que aporta el sector servicios es del $22 \%$; más aún, el dato correspondiente a las unidades económicas correspondientes al sector respecto del total nacional es del 37\% (Cuéntame INEGI, 2017).

La información previa expone la importancia del sector servicios para la economía en México, considerando tanto los empleos generados, la aportación al PIB nacional y el número de empresas existentes, por lo que la presente investigación se enfocará en dicho sector. De acuerdo al Sistema de Clasificación Industrial de América del Norte (SCIAN) el sector de servicios privados no financieros se subdivide de la manera mostrada en la Tabla 1 (INEGI, 2013b).

Tabla 1. Clasificación de los servicios privados no financieros.

\begin{tabular}{cl}
\hline Categoría SCIAN & Descripción \\
\hline $48-49$ & Transportes, correos y almacenamiento \\
51 & Información en medios masivos \\
53 & Servicios inmobiliarios y de alquiler de bienes muebles e intangibles \\
54 & Servicios profesionales, científicos y técnicos \\
56 & Servicios de apoyo a los negocios y manejo de desechos y servicios de \\
& remediación \\
61 & Servicios educativos \\
62 & Servicios de salud y de asistencia social \\
71 & Servicios de esparcimiento culturales y deportivos, y otros servicios recreativos \\
72 & Servicios de alojamiento temporal y de preparación de alimentos y bebidas \\
\hline
\end{tabular}

Fuente: Encuesta Anual de Servicios Privados No Financieros 2013.

A partir de la realización de un análisis del personal ocupado por los subsectores que conforman a los servicios en México (Banco de Información Económica, 2017), se ha elaborado la Figura 2. Como se puede apreciar, durante el periodo analizado (2008-2016) el indicador de personal ocupado que presentó un mayor incremento anual promedio dentro del sector de servicios privados no financieros fue el subsector de servicios de apoyo a los negocios y manejo de desechos y servicios de remediación con un 2.2\%. Lo 
anterior refleja que dicho subsector es el que ha aumentado más en cuanto a la cantidad de empleos que aporta al sector de los servicios.

Figura 2. Incrementos porcentuales anuales promedio en el personal ocupado en México correspondiente a cada subsector durante el periodo 2008-2016 del sector servicios.

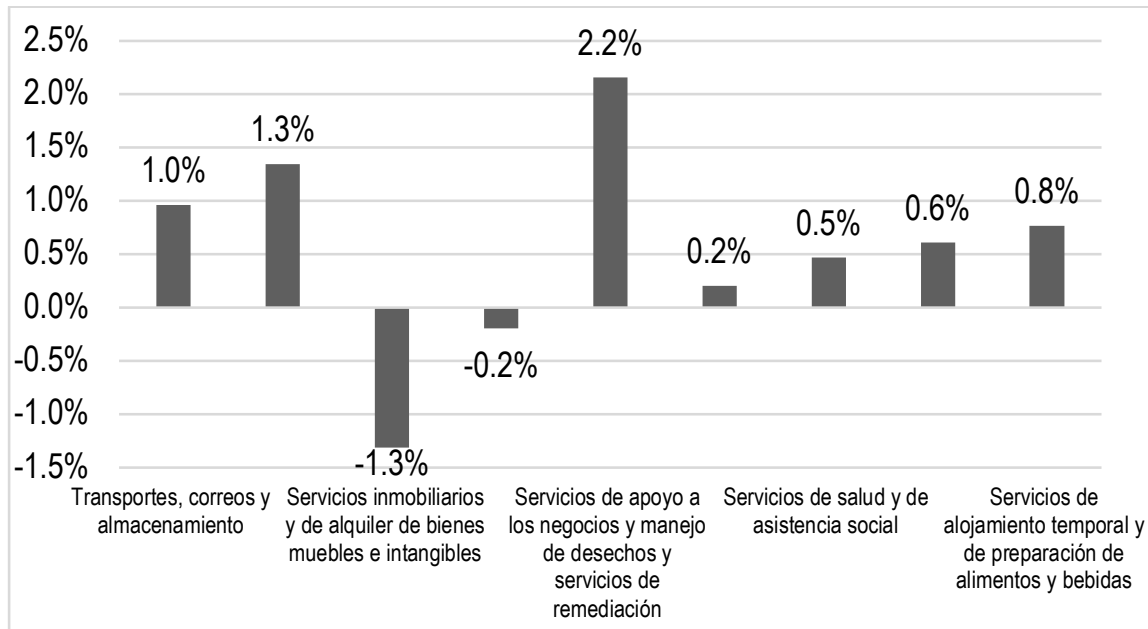

Fuente: Elaboración propia con base en: $\underline{\text { htpp://www.inegi.org.mx/sistemas/bie/ }}$

Además de lo anterior, es importante señalar que el dato correspondiente a incremento promedio anual de todo el sector fue únicamente de $0.88 \%$ (Banco de Información Económica, 2017) para el mismo periodo. Debido a estas razones se puede considerar al subsector de servicios de apoyo a los negocios y manejo de desechos y servicios de remediación como aquél de mayor importancia para la generación de empleos dentro del sector de los servicios en México, por lo que el presente trabajo de investigación se concentrará en dichas empresas de manera particular.

EI SCIAN (INEGI, 2013a) plantea las ramas correspondientes a los servicios de apoyo a los negocios y manejo de desechos y servicios de remediación que se presentan en la Tabla 2. 
Tabla 2. Clasificación de las ramas pertenecientes a los servicios de apoyo a los negocios y manejo de desechos y servicios de remediación por el SCIAN.

\begin{tabular}{ll}
\hline Código SCIAN & Actividad económica \\
\hline 5611 & Servicios de administración de negocios. \\
5612 & Servicios combinados de apoyo en instalaciones. \\
5613 & Servicios de empleo. \\
561510 & Agencias de viajes. \\
561520 & Organización de excursiones y paquetes turísticos para agencias \\
561590 & de viajes. \\
5616 & Otros servicios de reservaciones. \\
5617 & Servicios de investigación, protección y seguridad. \\
561710 & Servicios de limpieza. \\
561910 & Servicios de limpieza de inmuebles. \\
561920 & Servicios de empacado y etiquetado. \\
5621 & Organizadores de convenciones y ferias comerciales e \\
\hline
\end{tabular}

Fuente: Elaboración propia con base en:

http://www.inegi.org.mx/est/contenidos/proyectos/encuestas/establecimientos/terciario/easpnf/default_t.aspx

Al analizar las ramas mencionadas anteriormente es posible identificar que, más allá del valor económico de la producción generada, existen algunas de ellas que debido a las actividades que realizan, tienen un mayor impacto para la salud y el bienestar individual y social de las personas. La importancia concreta de las empresas pertenecientes a la rama de manejo de desechos y servicios de remediación radica en que son empresas cuyas actividades se encuentran enfocadas en mejorar las condiciones en las que se gestiona el impacto generado al medio ambiente por las actividades productivas.

El crecimiento poblacional es un fenómeno que incrementa de manera constante los retos para el Estado en cuanto a cobertura y eficiencia en la generación y distribución de bienes y servicios demandados por la población. Al existir un creciente número de personas es evidente que las necesidades que se necesitan satisfacer también son cada vez mayores. Con base en análisis realizados por la Organización para la Cooperación y el Desarrollo Económico (SEMARNAT, 2008) existe una relación entre el incremento del PIB y la capacidad de gasto de la población, y el aumento en el volumen de generación de residuos sólidos, asociado con la producción y consumo de bienes. De acuerdo a la definición proporcionada por SEMARNAT (2008), los 
residuos sólidos urbanos pueden definirse como: "aquellos generados en las casas habitación, que resultan de la eliminación de los materiales que utilizan en sus actividades domésticas, de los productos que consumen y de sus envases, embalajes o empaques; los residuos que provienen de cualquier otra actividad dentro de establecimientos 0 en la vía pública que genere residuos con características domiciliarias, y los resultantes de la limpieza de las vías y lugares públicos, siempre que no sean considerados por esta Ley como residuos de otra índole."

Con base en información de SEMARNAT (2008), presentada en la Figura 3, en México se generan 102,985 toneladas de residuos diarios, de los cuales son recolectados el $83.93 \%$ y son dispuestos en sitios de disposición final el $78.54 \%$. Dicho nivel de recolección pone de manifiesto la falta de cobertura que aún existe en el país para cubrir con dicho servicio de manera efectiva, de permite concluir un área de oportunidad importante para empresas nuevas y existentes que se enfoquen en la rama de manejo de desechos y servicios de remediación.

Figura 3. Información porcentual sobre los residuos sólidos urbanos en México.

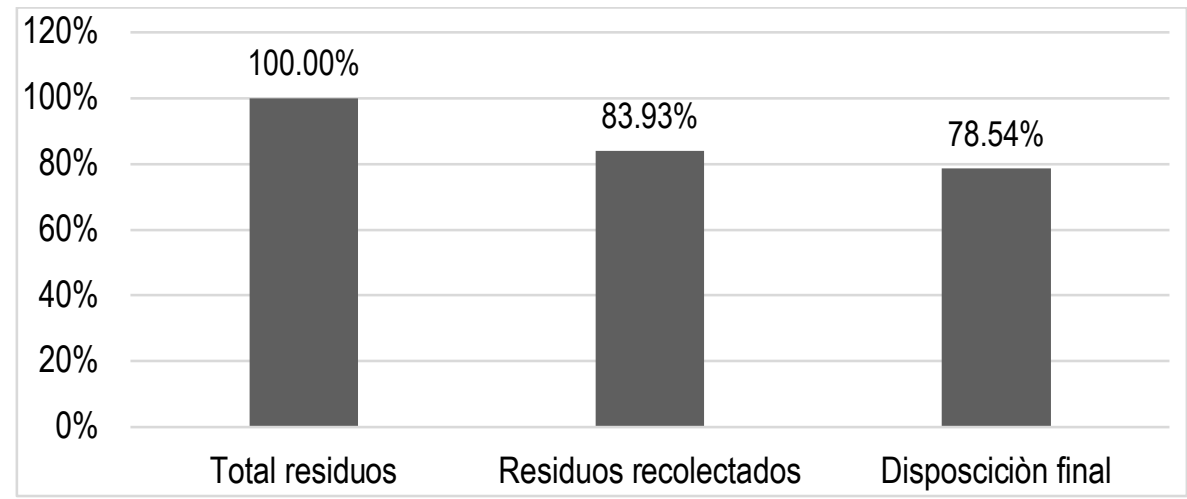

Fuente: Elaboración propia con base en:

https://www.gob.mx/semarnat/acciones-y-programas/residuos-solidos-urbanos-rsu

En el corto plazo se puede identificar un efecto positivo en la procuración de la salud de las personas al mejorar el medio ambiente en el que viven o trabajan, y en el largo plazo las operaciones de dichas empresas generar un equilibrio entre el crecimiento económico y un desarrollo 
sustentable que permita la perdurabilidad de los recursos naturales que son indispensables para la propia sobrevivencia humana. El Estado, a través de diversos organismos como la Secretaría de Medio Ambiente y Recursos Naturales se ocupa de prever problemas que se pudieran generar a este respecto y de establecer lineamientos y guías que permitan alcanzar una mejora en las prácticas sociales y empresariales sin comprometer los beneficios futuros, siempre enfocándose en el impacto que las actividades económicas pudieran tener en la salud y bienestar humano en general así como en el cuidado de animales, plantas y de los ecosistemas en los que habitan.

De acuerdo a los argumentos presentados anteriormente se puede concluir que las empresas pertenecientes a la rama de manejo de desechos y servicios de remediación dentro del sector servicios en México, además de presentar una importante área de oportunidad desde el punto de vista de inversión y emprendimiento, significan también una contribución a una causa social y ambiental con beneficios de largo plazo para un problema de interés público; por lo que cuentan con la pertinencia económica y social requerida para ser el objeto de estudio de la presente investigación.

EI SCIAN (INEGI, 2013b) describe a las empresas dedicadas al manejo de desechos y servicios de remediación: "unidades económicas del sector privado dedicadas principalmente a proporcionar servicios de manejo de residuos y desechos, y servicios de remediación a zonas dañadas por materiales o residuos peligrosos, o desechos no peligrosos, como recolección de residuos o desechos, tratamiento de residuos peligrosos, disposición de residuos o desechos, recuperación y clasificación de materiales reciclables no peligrosos, y rehabilitación y limpieza de zonas contaminadas. Incluye también: unidades económicas dedicadas principalmente a las actividades de saneamiento, como los servicios de limpieza de fosas sépticas, y al alquiler de sanitarios portátiles."

Como se planteó al inicio de la presente introducción, las empresas son los principales generadores del crecimiento económico para un país, de ahí la importancia de enfocar la disciplina de la valuación hacia dichas organizaciones permitiendo a los tomadores de decisiones empresariales una toma de decisiones más efectiva y oportuna.

Considerando lo anterior, Siu-Villanueva (1999) comenta que diversos elementos como: la gran cantidad de variables a considerar en los pronósticos 
financieros y la disminución en los niveles de utilidades, la creciente complejidad en los negocios en la actualidad, la variedad de características cualitativas que se deben considerar, el incremento en la competencia, la utilización de métodos confiables, así como las metodologías de valuación teórica, entre otros, han incidido en el incremento del valor agregado así como en la importancia de la valuación de empresas al considerar decisiones de inversión o desinversión en negocios.

De acuerdo a Caballer V. (1994) la valuación de empresas es "aquella parte de la economía cuyo objeto es la estimación de un determinado valor 0 valores de una empresa con arreglo a unas determinadas hipótesis, con vistas a unos fines determinados y mediante procesos de cálculo basados en información de carácter técnico y económico". Para su estudio, los métodos de valuación de empresas se pueden clasificar de manera general en: métodos de flujos de caja, contables y de mercado, considerando para su aplicación la cantidad y naturaleza de la información disponible.

Los métodos de flujos de caja, de acuerdo a Johnson \& Kuby (2004) consideran que el valor del negocio está dado por la sumatoria de los flujos de efectivo futuros que la empresa es capaz de generar, considerando el valor del dinero en el tiempo. Por su parte, Altair (2007), expone que para valuar una empresa a través de estos métodos es necesario realizar los siguientes puntos: a) determinar el número de periodos a analizar, b) realizar las proyecciones de los flujos de efectivo, c) estimar las tasas y datos de descuento, d) calcular la perpetuidad y el valor residual del negocio en marcha.

Para Barreneche \& Arenas (2007) los principales métodos contables son el de valor en libros y el valor en libros ajustado, en los cuales los costos de realización son descontados por las depreciaciones según sea correspondiente. Dicho enfoque de valuación considera asignarle a los activos el valor que se encuentre registrado en la contabilidad de la empresa y ajustarle con base en los cambios de valor correspondientes. La ventaja más destacada recae en la sencillez para comprenderlo y actualizarlo, considerando que la información requerida se encuentra en la propia contabilidad del negocio. Su principal desventaja consiste en que los resultados no son tan confiables debido a que se utiliza información estática, sin considerar el futuro, además de que tomar en cuenta la variable de depreciación en ocasiones no se ajusta a la realidad. 
Los métodos de mercado se basan en la determinación de diversas variables de comparación entre diversas empresas obtenidas de una gran cantidad de fuentes de información que conformen bases de datos confiables. Los valores son precios registrados de transacciones históricas que son consideradas homologables al valor del activo que se desea estimar. De acuerdo a Fernández (2007), algunos de los elementos más importantes que deben cumplir las empresas consideradas como comparables son: ser de un mismo país o región y/u operar en una misma zona geográfica, deben realizar la misma actividad que la empresa objetivo, tener un tamaño similar, tener una rentabilidad similar, tener unas mismas perspectivas de crecimiento de beneficios, tener una posición competitiva equivalente, entre otros.

Dentro de los métodos comparativos o de mercado, Ballestero (1971), propuso en su momento un método que aún hoy en día destaca por su sencillez y aplicabilidad para cualquier mercado del que se disponga la información suficiente, el denominado método de las dos betas; el cual parte de las siguientes hipótesis principales (Universidad Politécnica de Valiencia, 2011):

1. El valor de un bien económico es función de una variable independiente.

2. Tanto los valores de mercado de los bienes económicos como sus variables independientes presentan la forma de la distribución de probabilidad Beta.

3. Las funciones Beta se definen al conocer sus valores máximo, mínimo y moda.

La principal aportación del método consiste en el segundo punto, aquél en el que autor afirma que tanto los valores de mercado de los bienes económicos como sus variables explicativas siguen lo que se le llama como distribución beta.

Es de esta forma quela presente investigación se enfocará en empresas dedicadas al manejo de desechos y servicios de remediación; utilizando información de valores de compra-venta de empresas pertenecientes a dicha rama para establecer un modelo de valuación por medio del método de las dos betas que sea aplicable, representativo y útil para la toma de decisiones.

Por todo lo presentado anteriormente, la pregunta de investigación es: ¿es posible determinar el valor de una mipyme dedicada al manejo de desechos y servicios de remediación en México por medio del método de 
valuación de las dos betas? Consecuentemente, el objetivo de la investigación es: demostrar que es posible obtener el valor de una mipyme dedicada al manejo de desechos y servicios de remediación en México por medio del método de valuación de las dos betas.

A continuación se procederá a revisar los fundamentos teóricos básicosdel método de valuación de las dos betas.

\section{Marco Teórico}

De acuerdo a López (2006), la distribución Beta es utilizada con frecuencia en las denominadas técnicas PERT (Program Evaluation \& Review Techniques) para el control de proyectos. En dichas técnicas se supone que los tiempos de cada actividad que son parte de un proyecto son variables aleatorias que obedecen a la distribución Beta. Un ejemplo descriptivo de dicha función se presenta en la Figura 4.

Figura 4. Ejemplo gráfico de distribución de probabilidad Beta o de densidad.

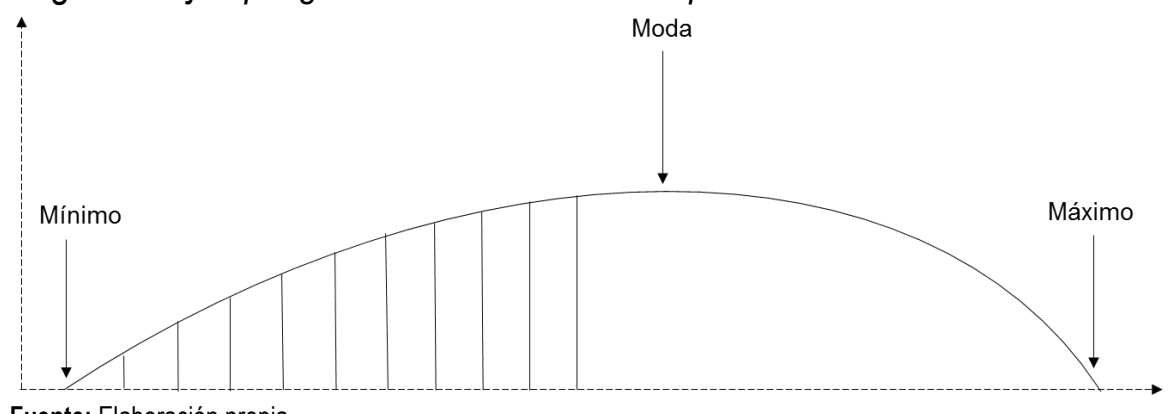

Fuente: Elaboración propia.

Como se puede observar, la distribución beta o función de densidad queda definida por un valor mínimo, moda y máximo; además de estar determinado en el eje positivo de las x's lo cual las hace funciones muy útiles para estudios económicos debido a que no pueden adoptar valores negativos.

La principal ventaja del método de valuación de las dos betas es que la información requerida es mínima (mínimo, máximo y moda del mercado al que pertenece el activo a valuar). Por su parte las desventajas son: se pueden calcular tantos valores como variables independientes se utilicen para su 
estimación, cada valor obtenido es proporcional a la variable empleada para su estimación y que el modelo presenta dificultad para emplear variables cualitativas.

De acuerdo al planteamiento propuesto por Ballestero (1973), el método de las dos betas se puede describir de la siguiente forma general (Universidad Politécnica de Valencia, 2011):

Los datos utilizados son mínimo (a), máximo (b) y moda (M).

Las hipótesis del modelo son:

$$
\begin{gathered}
p=h+\sqrt{2} \\
q=h-\sqrt{2} \\
p+q=2 \cdot h
\end{gathered}
$$

Para el cálculo de la variable $h$ se utiliza la Ecuación 2.

$$
h=\frac{\sqrt{2}(b-a)}{2 \cdot M-(a+b)}-1
$$

Con base en información del mercado de un activo determinado y de las características del mismo (valor máximo, mínimo y moda) se estima la forma de la función de distribución del Valor $V$ y de la variable independiente 0 explicativa $L f(V)$ y $g(L)$. En el activo objeto de la valuación se calcula el valor específico que alcanza el índice externo o variable explicativa $L_{x}$.

Figura 5. Distribución Beta de la variable independiente.

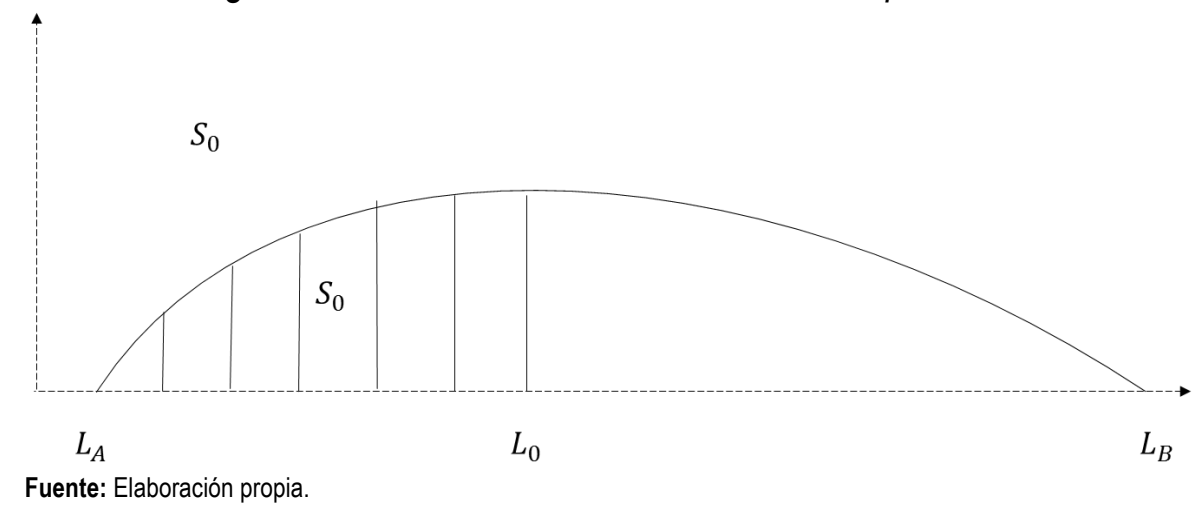

Se utiliza la fórmula presentada anteriormente correspondiente a la variable explicativa como se muestra en la Ecuación 3. 
donde:

$$
h=\frac{\sqrt{2} \cdot\left(L_{B}-L_{A}\right)}{\left[2 \cdot L_{M}-\left(L_{A}+L_{B}\right)\right]}
$$

$L_{A}=$ variable independiente inferior.

$L_{B}=$ variable independiente superior.

$L_{M}=$ variable independiente moda.

A partir de dicho valor $h$ se calculan los parámetros $p$ y $q$; mediante los cuales se obtiene el área que determina el activo a valuar dentro de la función beta de la variable independiente.

Figura 6. Distribución Beta de los valores de mercado.

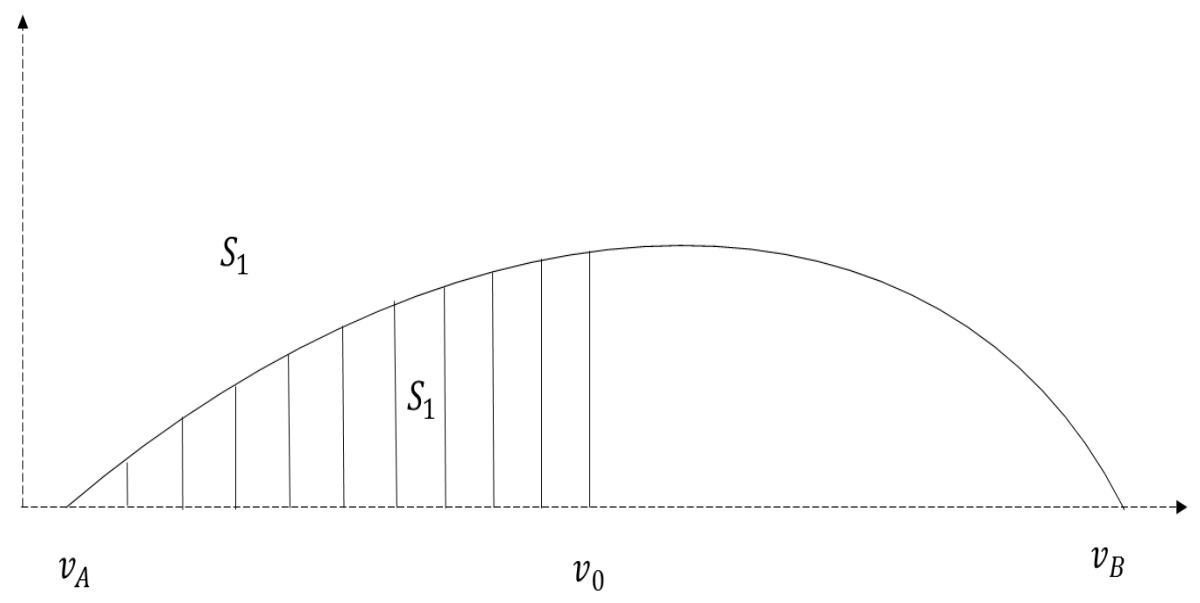

Fuente: Elaboración propia.

De manera similar, se utiliza la fórmula para estimar el valor $h$ del activo a valuar como se muestra en la Ecuación 4.

donde:

$$
h=\frac{\sqrt{2} \cdot\left(V_{B}-V_{A}\right)}{\left[2 \cdot V_{M}-\left(V_{A}+V_{B}\right)\right]}
$$

$V_{A}=$ valor inferior.

$V_{B}=$ valor superior.

$V_{M}=$ valor moda. 
Posteriormente se traslada al eje de ordenadas de la función de distribución de la variable explicativa $f(L)$. Al trasladar $L_{x}$ a la función beta de la variable explicativa, se divide a esa función en dos áreas; por lo que con el apoyo de tablas, de manera gráfica o por medio de aplicaciones informáticas se estima el valor de la superficie delimitada por la función $g(L)$ así como el punto $L_{x}$. A continuación, dicha área es trasladada a la función del valor $f(V)$; y el punto extremo de esa área nos indica el valor buscado.

Para la etapa del método en el que se traslada el área de la distribución beta de la variable independiente a la distribución beta del activo a valuar, originalmente se realizaba mediante el uso de tablas; hoy en día existen herramientas de software informático como la hoja de cálculo del programa Microsoft Excel que permiten realizar el cálculo de una manera mucho más sencilla.

Con base en Caballer \& Herrerías (2007) el principio básico que rige al método es el siguiente: si el indicador de calidad de un bien $i, x_{i}$, no supera al de otro bien $j, x_{j}$, el valor de mercado correspondiente al primer bien $i, y_{i}$, no superará el valor de mercado del bien $j, y_{j}$. A partir de este principio y supuesto conocidas las funciones de distribución de la variable aleatoria indicador, $X$, y la función de distribución de la variable aleatoria valor de mercado, $Y$, el valor de mercado, $y_{k}$, correspondientes al índice de calidad de un bien $k, x_{k}$, se obtiene igualando las dos funciones de distribución, esto es:

$$
F_{X}\left(X_{k}\right)=F_{Y}\left(X_{k}\right)
$$

donde:

$y_{k}=F_{Y}^{-1}\left(F_{X}\left(X_{k}\right)\right)$ será el valor de mercado del bien $\mathrm{k}$.

Como extensión a dicho método Romero (1977) comenta que en ciertos casos puede ser mejor utilizar una distribución de tipo triangular debido a que dicha función queda perfectamente determinada si se conocen la moda y valores extremos, caso que no ocurre en las distribuciones beta cuando no se han seleccionada con anticipación una particular entre ellas, además de que al utilizar la distribución triangular puede evitarse recurrir a tablas estadísticas y los errores cometidos son aproximadamente los mismos que con la distribución beta. 
Posteriormente han sido varios autores como: Caballer (1999), Herrerías, García \& Cruz (2001) y García, Cruz, \& García (2002), entre otros; los que han continuado con la realización de diversas aportaciones respecto al método original. De acuerdo a García, Trinidad \& García (2004) las aportaciones se pueden resumir en las siguientes líneas:

1. Aplicaciones prácticas de la metodología de las dos betas,

2. Extensión del método a diferentes distribuciones como: uniformes, trapezoidales, varianza constante, etc.,

3. Uso de dos o más índices implementando principios econométricos,

4. Generación de pruebas estadísticas para comparar la validez de las funciones de distribución utilizadas y

5. Procedimientos iterativos de valuación.

Sin embargo, como lo menciona Caballer (1998), la razón principal por la que se utiliza la distribución Beta y no otras funciones de probabilidad es que:

1. La variable de valor suele ajustarse de manera satisfactoria a una función de distribución Beta, debido a la forma de campana más o menos asimétrica que presentan los polígonos de las frecuencias de Valor en la realidad. Algo similar ocurre con la variable explicativa a elegir.

2. Aunque se dispongan de pocos datos para la valuación, en la práctica es frecuente poder encontrar:

a. El valor más bajo del mercado, que corresponde al límite inferior representado por los activos de la más baja calidad.

b. El valor más alto del mercado, que corresponde al límite superior representado por los activos de la más alta calidad.

c. El valor más frecuente de mercado, que corresponde a la calidad más común o la moda de la distribución. De manera similar, se considera que obtener los mismos datos de la variable independiente es de relativa facilidad.

Es importante mencionar que tanto la metodología original como sus variantes que utilizan diferentes funciones de distribución, han sido aplicadas principalmente para la valuación de terrenos agrícolas y activos inmobiliarios; por lo que el objetivo del presente trabajo es presentar un caso práctico realizado por medio de las dos distribuciones beta, contribuyendo a su uso en 
la determinacióndel valor de empresas, ampliando el campo de actuación de la valuación y brindando más herramientas para la toma de decisiones en los negocios.

\section{Método}

Habiendo presentado el procedimiento básico de la valuación por medio de las dos distribuciones beta, se procederá a calcular las estimaciones correspondientes, considerando como la muestra estadística a empresas que presenten las siguientes características:

1. Pertenecientes al sector servicios en México.

2. Enfocadas al subsector de servicios de apoyo a los negocios y manejo de desechos

3. Micro ( 1 - 10 personas) y pequeñas empresas ( 11 a 50 personas). Esta clasificación corresponde al sector servicios en México establecida por (Cuéntame INEGI, 2017).

4. De acuerdo en la subclasificación presentada previamente, se han elegido específicamente empresas que pertenezcan a la rama de:

a. Manejo de desechos y servicios de remediación.

Se han elegido micro y pequeñas empresas debido a que en conjunto representan el 99.2\% (INEGI, 2015) de unidades económicas pertenecientes al sector de servicios. Para la determinación del tamaño de la muestra, la estadística proporciona diversas herramientas para estimarla a partir de una población, error permitido y nivel de confianza dado. Tomando en consideración las características de la población analizada en el presente estudio, se determinó que la fórmula específica para calcular la muestra correspondiente es la de poblaciones finitas y variables de intervalo o razón (Johnson \& Kuby, 2004), la cual se presenta en la Ecuación 6. 


$$
n=\frac{Z^{2} N p q}{\left[e^{2}(N-1)\right]+\left[Z^{2} p q\right]}
$$

donde:

$\mathrm{N}=$ Población.

Z = Desviación estándar. Para un Nivel de Confianza del 95\% le corresponde un valor de 1.96 .

$\mathrm{e}=$ Error máximo permitido.

$p=$ Probabilidad de que suceda el evento.

$q=$ Probabilidad de que no suceda el evento.

Para la determinación de la población se ha considerado como un caso de estudio de 33 empresas representativas pertenecientes a la rama seleccionada. El número de 33 observaciones se ha determinado de acuerdo a un caso de estudio de empresas de las cuales se pudo obtener la información requerida por medio de un convenio con una empresa consultora en la Ciudad de Puebla, de la cual se omitirá su nombre para cubrir con el cumplimiento al contrato de confidencialidad correspondiente.

Debido a esto, la presente investigación se considera que utiliza un tipo de muestreo no probabilístico con las características de un muestreo intencional o selectivo (Bonilla-Castro \& Rodríguez, 2005), considerando que desde el inicio de la investigación ya se contaba con la información requerida. Sustituyendo los valores respectivos en la fórmula presentada se obtiene la Ecuación 7.

$$
n=\frac{1.96^{2}(33)(0.5)(0.5)}{\left[0.05^{2}(33-1)\right]+\left[1.96^{2}(0.5)(0.5)\right]}=30
$$

De esta forma, la muestra definitiva a analizar será de 30 micro, pequeñas y medianas empresas pertenecientes al sector de manejo de residuos y desechos en México. En correspondencia a la naturaleza de la investigación elaborada, se ha respetado la confidencialidad de las empresas tomadas como muestra, por lo cual no serán especificados nombres, productos o lugares, exclusivamente se analizaran los datos necesarios para los cálculos propuestos por la metodología elegida.

Es importante mencionar que las 30 empresas seleccionadas se encuentran operando dentro de la actividad económica del sector social, es 
decir que son: "ejidos, organizaciones de trabajadores, cooperativas, comunidades, empresas que pertenezcan mayoritaria o exclusivamente a los trabajadores $y$, en general, de todas las formas de organización social para la producción, distribución y consumo de bienes y servicios socialmente necesario." (Constitución Politica de los Estados Unidos Mexicanos, 2017); y que de acuerdo a (Ley de la Economia Social y Solidaria, 2013) "funcionan como un sistema socioeconómico creado por organismos de propiedad social, basados en relaciones de solidaridad, cooperación y reciprocidad, privilegiando al trabajo y al ser humano, conformados y administrados en forma asociativa, para satisfacer las necesidades de sus integrantes y comunidades donde se desarrollan, en concordancia con los términos que establece la presente Ley".

Además, la información utilizada fue obtenida a través de un convenio de colaboración con una empresa consultora ubicada en la Ciudad de Puebla, de la cual se omitirá su nombre por respeto al cumplimiento del contrato de confidencialidad correspondiente. A causa de la naturaleza de la investigación realizada, se puede establecer que se ha utilizado un tipo de muestreo no probabilístico con las características de un muestreo intencional o selectivo (Bonilla-Castro \& Rodríguez, 2005), debido a que ya se contaba con la información requerida para realizar la investigación desde los inicios del estudio.

De esta manera las conclusiones derivadas al final de la investigación podrán ser igualmente aplicables y representativas de empresas que se encuentren o no definidas como empresas con enfoque social.

\section{Resultados}

Los valores utilizados para los siguientes cálculos corresponden a las 30 empresas pertenecientes al mercado elegido de las cuales se obtuvo información, las cuales son consideradas como comparables u homologables a la empresa en particular de la que se desea determinar el valor.

De acuerdo al marco teórico y método presentados anteriormente, los datos obtenidos de la variable independiente 0 explicativa elegida,a partir de la investigación con fuentes primarias enfocada en el mercado de empresas dedicadas a la rama de manejo de desechos y servicios de remediación, se presentan en la Tabla 2. 
Tabla 2. Variable explicativa o independiente de la muestra de empresas analizada. Activos fijos en miles de pesos mexicanos.

\begin{tabular}{cc}
\hline Empresa & Activos Fijos \\
\hline 1 & $\$ 122$ \\
2 & $\$ 122$ \\
3 & $\$ 353$ \\
4 & $\$ 353$ \\
5 & $\$ 353$ \\
6 & $\$ 438$ \\
7 & $\$ 438$ \\
8 & $\$ 438$ \\
9 & $\$ 438$ \\
10 & $\$ 438$ \\
11 & $\$ 675$ \\
12 & $\$ 675$ \\
13 & $\$ 675$ \\
14 & $\$ 675$ \\
15 & $\$ 675$ \\
16 & $\$ 675$ \\
17 & $\$ 866$ \\
18 & $\$ 866$ \\
19 & $\$ 866$ \\
20 & $\$ 866$ \\
21 & $\$ 866$ \\
22 & $\$ 866$ \\
23 & $\$ 1,024$ \\
24 & $\$ 1,024$ \\
25 & $\$ 1,024$ \\
26 & $\$ 1,024$ \\
27 & $\$ 1,024$ \\
28 & $\$ 1,380$ \\
29 & $\$ 1,380$ \\
30 & $\$ 1,380$ \\
\hline
\end{tabular}

Fuente: Elaboración propia con base en el estudio realizado.

La variable independiente seleccionada se refiere los Activos Fijos de las empresas, es decir, aquellos activos con vida útil de más de un año y que se utilizan en las operaciones de la empresa, principalmente son: edificios, terrenos, mobiliario y equipo, maquinaria, vehículos (Meza, 2007).

Es importante clarificar que los datos han sido redondeados para fines de la presente investigación, sin que ello afecte en una gran proporción la 
información original. A partir de dicha base de datos se puede obtener la siguiente información requerida por la metodología propuesta:

Tabla 3 Límite inferior, límite superior y moda de los datos recabados por le investigación correspondientes a la variable independiente. Valores en miles de pesos mexicanos.

\begin{tabular}{cc}
\hline Límite & Variable Explicativa \\
\hline Mínimo & $\$ 122$ \\
Moda & $\$ 675$ \\
Máximo & $\$ 1,380$ \\
\hline
\end{tabular}

Fuente: Elaboración propia con base en el estudio realizado.

El paso siguiente consiste en estimar los valores de $h, p$ y $q$, para lo cual se utilizan las fórmulas respectivas:

$$
h=\frac{\sqrt{2}(\text { máximo-mínimo })}{2 \cdot \operatorname{moda}-(\text { máximo }+ \text { mínimo })}-1
$$

sustituyendo:

$$
h=\frac{\sqrt{2}(\$ 1,380-\$ 122)}{2 \cdot \$ 675-(\$ 1,380+\$ 122)}-1=10.704
$$

De esta forma queda estimado el dato de la variable $h$ en 10.704. De acuerdo a la metodología propuesta y dado que la moda es menor a la media de los datos de la variable independiente, el factor $p$ se obtiene con la aplicación de la Ecuación 9.

$$
p=h-\sqrt{2}
$$

El resultado de $p$ estimado resulta ser de: 9.290. Con base en los fundamentos del método de las dos betas y considerando que la moda es menor a la media de los datos de la variable independiente, el factor $q$ se calcula por medio de la Ecuación 10.

$$
q=h+\sqrt{2}
$$


El dato obtenido para la variable $q$ es $=12.119$. Una vez que se cuenta con los valores mínimo y máximo, así como los factores $h, p$ y qde la variable independiente se procede a estimar el área correspondiente dentro de la función de distribución beta que le correspondería al valor de la variable independiente perteneciente a la empresa que deseamos valuar. Lo anterior se realiza por medio de la función de distribución beta dentro del software informático Microsoft Excel de la siguiente forma:

$$
=\operatorname{DISTR} \cdot \operatorname{BETA}\left(L_{X}, p, q, L_{A}, L_{B}\right)=\text { Área }
$$

donde:

$L_{X}=$ variable independiente de la empresa a determinar su valor.

$L_{A}=$ variable independiente mínima.

$L_{B}=$ variable independiente máxima.

$p=$ factor $p$ estimado.

$q=$ factor $q$ estimado.

De esta forma, el área estimada obtenida es de $=$, lo cual representa al área que la variable independiente de la empresa a valuar define dentro de la función beta de la variable independiente del mercado al que pertenece la empresa, en este caso de la rama dedicada al manejo de desechos y servicios de remediación.

En seguida se procede a calcular las variables $h, p$ y $q$ correspondientes a la variable dependiente, es decir, el valor de las empresas consideradas como muestra.

Tabla 4. Límite inferior, límite superior y moda de los datos recabados por le investigación correspondientes a la variable dependiente. Valores en miles de pesos mexicanos.

\begin{tabular}{cc}
\hline Límite & Valor de compra-venta \\
\hline Mínimo & $\$ 153$ \\
Moda & $\$ 813$ \\
Máximo & $\$ 1,655$ \\
\hline
\end{tabular}

Fuente: Elaboración propia con base en el estudio realizado. 
Una vez más se utilizan las fórmulas respectivas:

sustituyendo:

$$
h=\frac{\sqrt{2}(\text { máximo-mínimo })}{2 \cdot \text { moda-(máximo+mínimo })}-1
$$

$$
h=\frac{\sqrt{2}(\$ 1,655-\$ 153)}{2 \cdot \$ 813-(\$ 1,655+\$ 153)}-1=10.671
$$

Así, queda calculado el dato de la variable h en 10.671. Con base en la metodología empleada y considerando que la moda es menor a la media de los datos de la variable independiente, el factor $p$ se obtiene utilizando la Ecuación 12.

$$
p=h-\sqrt{2}
$$

El resultado de $p$ estimado resulta ser de: 9.257. Tomando en cuenta los fundamentos del método de las dos betas y dado que la moda es menor a la media de los datos de la variable independiente, el factor $q$ se calcula por medio de la Ecuación 13.

$$
q=h+\sqrt{2}
$$

El dato obtenido para la variable $q$ es $=12.085$. Una vez que se conoce el área de la distribución beta correspondiente a la variable independiente definida por el activo a valuar, así como los factores $h, p$ y $q$ correspondientes a la variable dependiente de las empresas muestra de la investigación, se procede a trasladar dicha área a la segunda distribución beta, es decir a aquella correspondiente a la información de la variable dependiente. Dicho paso se realiza con el apoyo de Microsoft Excel como se muestra a continuación:

$$
\left.=\text { DISTR.BETA.INV(Área,p,q, } V_{A}, V_{B}\right)=V_{X}
$$

donde:

Área = área de la distribución beta estimada.

$p=$ factor $p$ estimado.

$q=$ factor $q$ estimado.

$V_{A}=$ variable dependientemínima.

$V_{B}=$ variable dependientemáxima.

$V_{X}=\$ 1,048.53$. 
El resultado obtenido de dicha función representa el valor de la variable dependiente correspondiente a la distribución beta de valores dependientes definida por el área calculada a partir de la función beta de la variable independiente; es decir es el valor de la empresa objeto de estudio.

\section{Conclusiones}

Debido a la dinámica y contexto mundial en la actualidad, la valuación de empresas se consolida como una herramienta de gran importancia para la toma de decisiones empresariales; por lo que el presente trabajo presenta un caso práctico de valuación de una mipyme por medio de una metodología novedosa en el ámbito empresarial. Especificamente, el sector de servicios privados no financieros en México es de gran relevancia para la economía nacional debido al personal ocupado que ofrece, de manera particular, el subsector de servicios de apoyo a los negocios y manejo de desechos y servicios de remediación ha presentado un incremento relevante durante al menos los últimos ocho años. De manera concreta, la rama de actividad correspondiente a empresas dedicadas al manejo de desechos y servicios de remediación dentro del subsector de servicios de apoyo en México constituye un área de gran relevancia tanto en el aspecto económico como en el social y medioambiental, por lo que la presente investigación se enfocó en proponer un modelo de valuación para dicha empresas.

Por otro lado, la metodología de valuación de las dos betas fue originalmente propuesta por el Profesor Enrique Ballestero perteneciente a la Universidad Politécnica de Valencia y ha tenido diversas aplicaciones y variantes enfocadas principalmente a la valuación de bienes inmuebles. El presente trabajo busca utilizar dichos principios originales y aplicarlos para un caso de valuación de empresas. Debido a que es un método comparativo o de mercado, se utilizó información referente a valores reales de empresas similares y comparables del mercado al que pertenece la empresa elegida, por lo que mediante la investigación con fuentes primarias de información, se obtuvieron los datos correspondientes a la variable elegida como independiente. De esta forma el valor de la empresa a valuar puede ser inferido por medio del análisis respectivo y las conclusiones se consideran aplicables a otras empresas homologables. 
Para la conformación de la base de datos requerida para la metodología propuesta, se consideró como caso de estudio a 33 micro, pequeñas y medianas empresas dedicadas al manejo de desechos y servicios de remediación en México, las cuales se considera presentaron características atribuibles a empresas con enfoque social. La información se obtuvo a partir de un convenio con una empresa consultora de la Ciudad de Puebla, a partir de dicha población se estimó una muestra estadística de 30 empresas para realizar las estimaciones respectivas.

Se eligió utilizar como variable independiente o predictora del modelo al rubro contable denominado como Activos Fijos, debido a que se considera una variable representativa del valor de una empresa, específicamente de la rama elegida; además de la facilidad para obtener dicho dato de cada una de las empresas consideradas en la muestra selectiva realizada. Atendiendo a los pasos requeridos por el método, se utilizaron los datos de mínimo, máximo y moda para estimar los factores $h, p$ y $q$ de la variable independiente para obtener la primera distribución beta; se obtuvo el área delimitada por la variable independiente de la empresa a valuar. Posteriormente se realizaron las estimaciones respectivas para determinar con el mínimo, máximo y moda para calcular los factores $h, p$ y $q$ de la variable dependiente y así conocer la segunda distribución beta. Finalmente se trasladó el área de la primera distribución a la segunda, y con ello se pudo determinar que el valor de la empresa objeto del caso de estudio es de $\$ 1,048.53$, expresado en miles de pesos mexicanos.

El resultado final obtenido presenta una lógica económica debido a que, considerando que el los activos fijos son mayores que la moda de la muestra,el valor de la empresa objeto de estudio es también mayor que la moda de valores de la muestra. Los tomadores de decisiones que busquen realizar o incrementar la inversión en dicha rama del sector servicios, deberán considerar las presentes conclusiones con el objetivo de alcanzar resultados financieros óptimos.Es de esta forma que se ha presentado un caso de estudio cuya metodología es aplicable a empresas de la rama y tamaños seleccionados pertenecientes al sector de servicios privados no financieros en México, lo cual representa una aportación para la generación de conclusiones pertinentes para los tomadores de decisiones en relación al valor de sus empresas e inversiones. 
De esta forma, tanto la pregunta de investigación, como el objetivo del presente estudio se cumplen al demostrar que es posible determinar el valor de una micro, pequeña y mediana empresa dedicada al sector de manejo de desechos y servicios de remediación en México utilizando el método de valuación de las dos betas, perteneciente al enfoque de mercado al ser aplicado a empresas.

Investigaciones posteriores deberán realizar estudios que permitan conocer el valor de empresas de diferentes subsectores y ramas de la economía, de empresas de diferentes tamaños, así como del uso de otros factores como variables independientes; además de la comparación de los resultados respectivos. De esta forma queda propuesta una metodología de valuación de empresas diferente a lo que generalmente se utiliza en la actualidad, validando la aportación científica del presente trabajo de investigación.

\section{Referencias}

ALTAIR (2007). Valoración de empresas por flujos de caja descontados, Valencia: ALTAIR consultores.

Ballestero, E. (1971). Sobre la valoración sintética de tierras y un nuevo método aplicable a la concentración parcelaria, Revista de Economía Política, 57, 225-238.

Ballestero, E. (1973). Nota sobre un nuevo método rápido de valoración, Revista de Estudios Agrosociales, 85, 75-78.

Banco de México. (2017). Glosario de términos. Recuperado el Marzo de 2017, de http://www.banxico.org.mx/divulgacion/glosario/glosario.html

Barreneche, L. \& Arenas, J. (2007). Especialización en finanzas y mercado de capitales. Dirección de educación avanzada. Medellín: Universidad de Medellín.

Bonilla-Castro, E. \& Rodríguez, P. (2005). Más allá del dilema de los métodos. Bogotá: Norma.

Caballer, V. (1994). Métodos de valoración de empresas. Madrid: Pirámide.

Caballer, V. (1998). Valoración agraria. Teoría y práctica. $4^{a}$ Ed. Madrid: Mundi-Prensa.

Caballer, V. (1999). Valoración de árboles, frutales, forestales, medioambientales, ornamentales. Madrid: Ediciones Mundi-Prensa.

Caballer, V. \& Herrerías, R. (2007). Tasación y valoración. Situación actual y perspectiva de futuro, Estudios de Economía Aplicada, 25(1), 23-48.

Constitución Politica de los Estados Unidos Mexicanos. (2017). Artículo 25. México.

Cuéntame INEGI. (2017). Parque Económico. Obtenido de Servicios: http://cuentame.inegi.org.mx/economia/parque/Servicios.html 
Fernández, P. (2008). Métodos de valoración de empresas. Documento de investigación. Barcelona: IESE Business School.

García, J., Cruz, S. \& García, L. B. (2002). Generalización del método de las dos funciones de distribución (MDFD) a familias beta determinadas con los tres valores habituales. Análisis, selección y control de proyectos y valoración. En Actas de la III Reunión Científica ASEPELT: Análisis, Selección, Control de Proyectos y Valoración. Murcia: Servicio de publicaciones de la Universidad de Murcia, 89-113.

García, J., Trinidad, J. E \& García, L. B. (2004). Valoración por el método de las dos funciones de distribución: cómo seleccionar la mejor distribución. XVIII Reunión Asepelt, León: Asociación Internacional de Economía Aplicada.

Herrerías, R., García, J. \& Cruz, S. (2001). II modello probabilistico trapezoidale nel metodo delle due distribución della teoria generale de valutazioni. "Genco Rurale", Rivista de Scienze Ambientali, 64, 3-9.

INEGI (2013a). Encuesta anual de servicios privados no financieros 2013. Aguascalientes: Instituto Nacional de Estadística y Geografía.

INEGI (2013b). Sistema de Clasificación Industrial de América del Norte. Aguascalientes: Instituto Nacional de Estadística y Geografía.

INEGI (2014). Censos económicos 2014. Resultados definitivos. Aguascalientes: Instituto Nacional de Estadística y Geografía.

INEGI (2015). Minimonografías: Micro, pequeña, mediana y gran empresa, Censos económicos 2014. Aguascalientes: Instituto Nacional de Estadística y Geografía.

INEGI (2017). Cobertura temática. Servicios. Banco de Información Económica. Aguascalientes: Instituto Nacional de Estadística y Geografía. Recuperado el Abril de 2017, de http://www.inegi.org.mx/sistemas/bie/

Johnson, R., \& Kuby, P. (2004). Estadística elemental. Lo esencial. Cd. México: Thomson Learning.

Ley de la Economia Social y Solidaria. (2013). Artículo 3. México.

López, R. (2006). Cálculo de probabilidades e inferencia estadística. Caracas: Universidad Católica Andrés Bello.

Meza, C. (2007). Contabilidad. Análisis de cuentas. San José de Costa Rica: Universidad Estatal a Distancia.

Romero, C. (1977). Valoración por el método de las dos distribuciones beta: una extensión. Revista de Economía Política, 75, 47-62.

SEMARNAT. (2008). Programa Nacional para la Prevención y Gestión Integral de Residuos. México: Secretaría de Medio Ambiente y Recursos Naturales.

SEMARNAT. (2017). Residuos sólidos urbanos y de manejo especial. (S. d. Naturales, Ed.) Recuperado el Junio de 2017, de https://www.gob.mx/semarnat/acciones-yprogramas/residuos-solidos-urbanos-rsu

Siu-Villanueva, C. (1999). Análisis y evaluación de proyectos de inversión para bienes de capital. Cd. de México: Instituto Mexicano de Contadores Públicos.

Universidad Politécnica de Valencia. (2011). Grupo de Valoración y Decisión Multicriterio. Recuperado el Junio de 2017. 